\title{
论 文
}

\section{血管舒张因子 NO 在神经-血管偶联过程中扩散动 力学的仿真建模}

\author{
杨勇 ${ }^{\mathbb{Q}}$ ，王明来 ${ }^{\circledR}$, 范影乐 ${ }^{\circledR}$, 郑篗祥 ${ }^{\circledR *}$ \\ (1) 杭州电子科技大学, 生物医学工程系, 杭州 310018 ; \\ (2) 浙江大学, 生物医学工程与仪器科学学院, 杭州 310028 \\ * 联系人, E-mail: zxx@mail.bme.zju.edu.cn \\ 收稿日期: 2010-01-26; 接受日期: 2010-02-25 \\ 国家自然科学基金(批准号: 30770685)资助项目
}

\begin{abstract}
摘要神经-血管偶联机制至今还没有完全被阐明. 对脑微循环的研究表明, 位于皮层内的 微动脉的舒张代表着神经-血管偶联过程中的最初血流响应机制. 一氧化氮(NO)被认为是介导 微动脉舒张的最重要因子之一, 为了探讨 $\mathrm{NO}$ 在微动脉舒张过程中作为关键因子的作用, 本文 开展了基于大脑功能柱水平, 由功能刺激产生的 $\mathrm{NO}$ 在神经-血管偶联过程中扩散动力学的时 空模式的仿真建模研究. 在大脑功能柱形态分析的基础上, 建立 NO 扩散数学模型. 应用该模 型, 清晰地阐述了由功能刺激产生的 NO 在时间维度和空间维度的扩散过程. 计算机仿真结果 表明, 由功能刺激产生的 NO, 其扩散主要被限制在功能柱内. 因此, NO作用的影响区域也就被 限制在功能柱内. 在时间维度上, $\mathrm{NO}$ 信号大约维持 $1 \mathrm{~s}$ 左右. 本研究从四维时空角度探讨由功 能刺激产生的血管舒张因子的响应模式, 为最终阐明神经-血管偶联机制提供了一种新的途径.
\end{abstract}

\section{关键词 \\ 一氧化氮 时空动力学 功能柱 神经血管偶联} 动脉舒张 神经-血管偶联(neurovascular coupling)即由神经 活动导致该局部区域脑血流量增加, 其机制目前还 未被完全阐明. 从动力学的角度分析, 神经-血管偶 联的血流响应过程是一个具有空间扩散项的时空过 程, 此响应过程是由被刺激神经元产生的介导因子 诱发而产生. 因此, 要充分理解神经-血管偶联机制, 必须探讨介导因子的时空动力学. 到目前所研究过 的介导因子当中, 一氧化氮(nitric oxide, NO) 已被认 为是介导微动脉舒张的最重要因子之一 ${ }^{[1]}$, 其介导作 用已得到不同实验的支持, 如不同脑区中的神经型 一氧化氮合酶(neuronal nitric oxide synthase, nNOS) 的免疫组化研究 ${ }^{[2]}$ 一氧化氮合酶抑制剂抑制脑血流
的药理学研究 ${ }^{[3]}$ 及在小脑局部脑区血流灌注的功能 成像研究等 ${ }^{[4]}$. 然而, 由于方法和技术的限制, 目前 仍难以对神经-血管偶联过程中 NO 扩散的时空动力 学进行在体实验动物研究.

对于像神经-血管偶联这种复杂现象，应用计算 机进行仿真模拟是有效的方法之一. 目前已有一些 有关在神经系统中 NO 如何进行扩散的理论建模研 究 ${ }^{[5 \sim 9]}$. 在最近的仿真建模研究中, Philippides 等人 ${ }^{[10]}$ 探讨了由神经丛(plexus)产生的 NO 作为合作信号 (cooperative signaling)扩散的时空动力学过程. 神经 丛存在脊椎动物及无脊椎动物中, 是由神经纤维组 成的蘑菇体状结构. 根据仿真结果, 他们推测神经丛

英文版见: Yang Y, Wang M L, Fan Y L, et al. Modeling columnar spatiotemporal dynamics of nitric oxide as a primary controlling element of arterioles dilation during neurovascular coupling. Sci China Life Sci, 2010, doi: 10.1007/s11427-010-0064-x 
这种大脑皮层形态的功能意义可能是参与脑血流的 调节. 然而, 脑解剖研究表明, 大脑皮层神经纤维不 与微动脉直接接触 ${ }^{[1]}$. 因此, 神经丛结构的功能是否 是血流量调节的一种形态学上的适应需进一步探讨.

实际上对哺乳动物而言, 大脑皮层结构更基本 的特点是功能柱结构 ${ }^{[12,13]}$. 大脑功能柱不仅在形态 上，而且在构成上也不同于神经从结构. 如神经从主 要由直径小于 $1 \mu \mathrm{m}$ 的神经纤维构成, 而大脑功能柱 构成非常复杂, 包括神经胶质细胞和神经元(通常神 经元在水平方向按层分布)及血管网络 (一般于大脑 表面垂直分布). 对脑微循环的研究表明, 调节进入 毛细血管网血流量的基本功能单位是微动脉. 因此, 位于皮层内的微动脉的舒张代表着神经-血管偶联过 程中的最初血流响应机制. 由于微动脉的分布和功 能柱密切相关, 因此, 研究神经-血管偶联机制, 必须 直接在功能柱水平进行研究.

本文为研究 $\mathrm{NO}$ 在功能柱水平的扩散动力学过 程, 首先建造了一个基于大脑功能柱形态的 $\mathrm{NO}$ 扩散 物理模型. 并作基本假设: 当功能柱被功能性刺激激 活后, 在柱内将产生一个 $\mathrm{NO}$ 脉冲; 产生的 NO 扩散 作用于周围动脉; 一旦 $\mathrm{NO}$ 浓度达到阈值( $\mathrm{NO}$ 使动脉 平滑肌细胞舒张的最低浓度), 在功能柱内或者附近 的动脉将有效地舒张平滑肌细胞, 并随后扩张动脉 以增加血液流量. 应用该模型, 从仿真角度清晰地阐 述了由功能刺激产生的 NO 在时间维度和空间维度 扩散的过程.

\section{1 材料与方法}

\section{1 模型的生理与解剖学基础}

激活的大脑皮质功能柱的几何结构被作为一种 物理模型来模拟.

(1) 功能柱的几何学. 解剖学上, 皮质功能柱的 直径大小在 300 600 $\mu \mathrm{m}$ 不等. 基于 Hubel 与 Wiesel ${ }^{[12]}$, Paxinos 与 Watson ${ }^{[14]}$ 的观察, 功能柱在视觉皮层区域 组成更大的结构单位, 称为超柱(hypercolumns). 在大 鼠脑皮层, 一个典型的超柱有一个长方体, 其底面是 边长约 $1000 \mu \mathrm{m}$ 的正方形, 深度约为 $1800 \mu \mathrm{m}$. 基于 大鼠视觉超柱的解剖学分析, 构建了一个典型超柱 的几何图形(图 1(A)).
(2) 氮能神经元(nitrergic neurons)在功能柱内的 扩散. 氮能神经元是含神经型一氧化氮合酶的神经 元, 氮能神经元在刺激状态下产生 NO. 因此, 功能 柱的几何结构被确定后, 另一个必须确定的先决条 件是 NO 在柱内的浓度分布如何? 这取决于氮能神 经元在功能柱内的分布情况. nNOS 的免疫细胞学研 究表明, 含有 NOS 的神经元稀疏地分布在大脑皮层, 比例约为 $2 \%^{[11]}$. 目前尚不清楚稀疏的氮能神经元是 否均匀地分布在柱内. 但近期的一项关于 NO 在大鼠 大脑不同皮层分布的研究指出, 产生 $\mathrm{NO}$ 的皮层间差 异只存在于产后早期阶段, 在成熟的大脑没有皮层 间差异 ${ }^{[15]}$. 因此, 认为整个矩形超柱有着均匀 NOS 分布是合理的.

(3) NO 作为神经-血管偶联中动脉扩张的一个主 要控制因子. 大脑由血管网络供给养分和氧气. 软脑 膜表面动脉在大脑表面水平分布, 皮质动脉 (由软膜 动脉分枝产生)从大脑表面垂直进入皮质柱并分支成 许多小动脉、微动脉和前毛细血管小动脉, 最后形成 一个高度吻合的大脑毛细血管网 ${ }^{[16]}$. 据 Woolsey 等 人 $^{[17]}$ 的研究, 一个功能柱内至少有几个皮质动脉. 因 此, 解剖学上, 神经元和柱内的动脉是相互联系的. 一旦产生 NO, 将迅速扩散作用于动脉中的平滑肌细 胞, 使平滑肌能够舒张. 因此, 认为 $\mathrm{NO}$ 是神经-血管 偶联中动脉扩张的一个主要控制因子. 神经元和动 脉在功能柱内的分布见图 1(B).

\section{$1.2 \mathrm{NO}$ 扩散的控制方程}

在激活状态下, 功能柱内进行 NO 的生物合成, 并在柱内外自由地扩散. 为描述扩散的动态过程, 使 用以下修改过的扩散方程 ${ }^{[18]}$ :

$$
\begin{aligned}
& \frac{\partial C\left(\begin{array}{l}
r \\
x
\end{array}, t\right)}{\partial t}-D\left(\frac{\partial^{2} C\left(\begin{array}{l}
r \\
x
\end{array}, t\right)}{\partial x^{2}}+\frac{\partial^{2} C\left(\begin{array}{l}
r \\
x
\end{array}, t\right)}{\partial y^{2}}+\frac{\partial^{2} C\left(\begin{array}{l}
r \\
x
\end{array}, t\right)}{\partial z^{2}}\right) \\
& =-\operatorname{Sink}\left(\begin{array}{l}
r \\
x
\end{array}\right) g C\left(\begin{array}{c}
r \\
x
\end{array}, t\right)+S\left(\begin{array}{l}
r \\
x
\end{array}, t\right),
\end{aligned}
$$

在该方程中, $C(\vec{x}, t)$ 是点 $\vec{x}$ 在时刻 $t$ 的浓度, $D$ 是扩 散系数 ${ }^{[7,9]}$. 函数 $S(\vec{x}, t)$ 代表神经元被激活时柱内 $\mathrm{NO}$ 合成的强度, 定义为每秒产生的 NO 浓度(单位: mol. 单位体积 $\left.{ }^{-1} \cdot \mathrm{s}^{-1}\right)$. 在仿真过程中, 假设神经元均匀分 布在柱内, 从而 $S(\vec{x}, t)$ 与特定的结构和位置无关. 因 此, $S(\vec{x}, t)=S(t)$. 
式子 $\operatorname{Sink}(\vec{x}) g C(\vec{x}, t)$ 是一个失活的函数，用于 表征各种氧化反应和结合血红素过程中损失的 NO. 由于认定神经元是均匀分布在柱内的, 故可以假设 $\operatorname{Sink}(\vec{x})=k$.

假设 NO 的衰减是一级反应，从而得到:

$$
t_{1 / 2}=\frac{\ln 2}{k} \text { equ. } 2,
$$

$t_{1 / 2}$ 被定义为 $\mathrm{NO}$ 的半衰期.

\section{3 计算方法}

使用有限元法进行仿真模拟，该方法已在数值

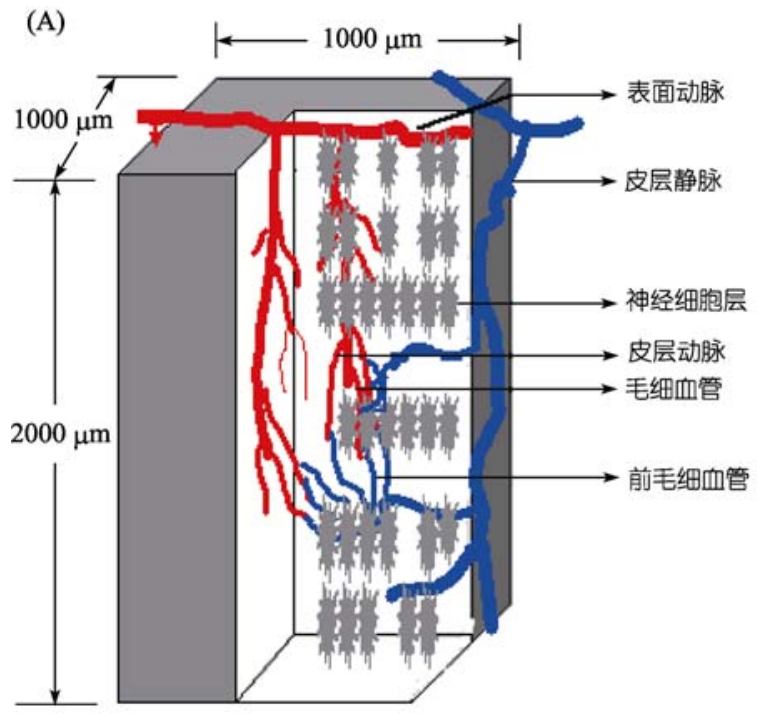

(B)

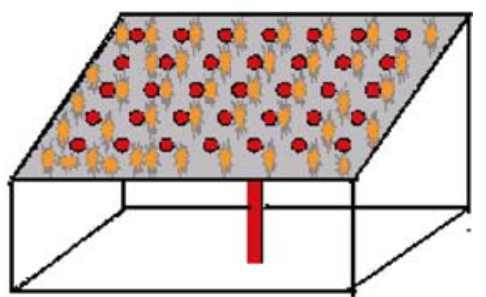

(C)

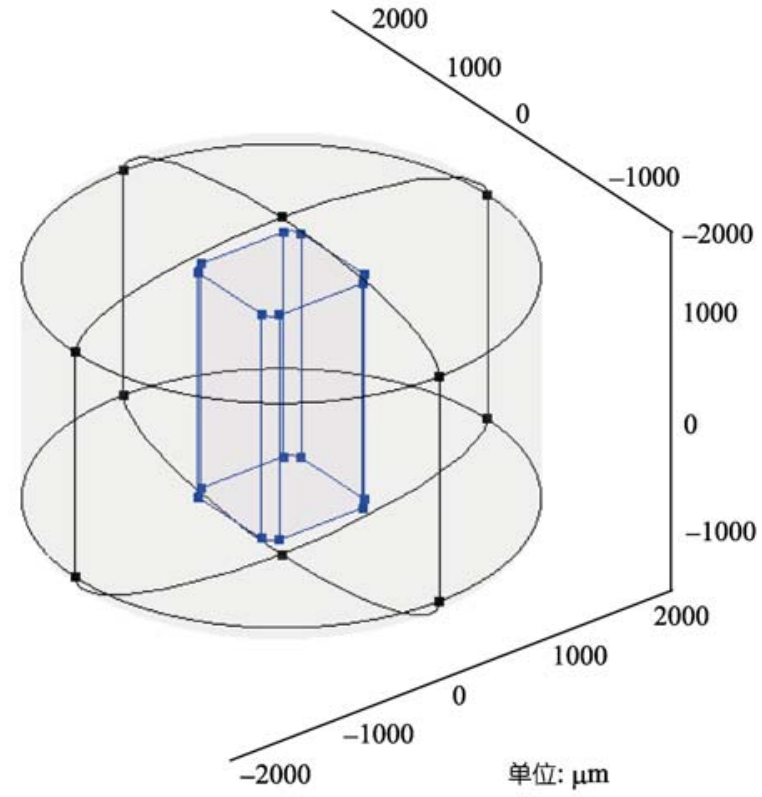

图 1 物理模型的描述

(A) 皮层超柱的几何结构和血管层次分布. 灰色矩形块代表超柱结构(白色为其剖面). 加粗的不同的红色和蓝色线条代表了各种动脉和 静脉分支, 其中分别包括软膜动脉、皮质动脉、皮质微动脉、皮质静脉、前毛细血管和毛细血管区带. 灰色星点代表在超柱状体内神经 元的层分布; (B) 神经元和动脉分布的功能柱的一部分; (C) 物理模型结构 
计算的说明中介绍 ${ }^{[19]}$.

在计算过程中, 包括网格和数值分化与整合，在 以相对精度为 $1 \%$ 的偏微分方程处使用执行 Comsol Femlab(www.comsol.com)来完成.

初始和边界条件设定如下:

（1）物理模型的结构显示在图 1(C).

(2) NO 初始浓度. NO 初始浓度(或 NO 阈值)计 算机模拟必须考虑的重要参数. 但是, NO 在体内的 浓度从 $1 \mu \mathrm{mol} / \mathrm{L}$ 到 $1 \mathrm{nmol} / \mathrm{L}$ 在已报道的文献中一直 具有争议 ${ }^{[20,21]}$. 本模型关注激活状态和稳定状态中 不同 $\mathrm{NO}$ 浓度数量级的变化. 例如, 假设在激活状态 $\mathrm{NO}$ 的浓度是稳定状态的 2 3 个数量级 (如果假定 NO 在稳定状态是 $1 \mathrm{nmol} / \mathrm{L}$, 其值在激活状态可能是 $100 \sim 1000 \mathrm{nmol} / \mathrm{L}$ ). 由于在浓度的数量级是独立的, 所以, NO 的浓度无论是在纳摩尔级别或是在微摩尔 级别都不会影响建模. 近期研究表明, NO 可能在纳 摩尔级 ${ }^{[22]}$. 假设 NO 在脑组织中的阈值(最低浓度)为 $1 \mathrm{nmol} / \mathrm{L}$.

(3) 当刺激产生, 假设 NO 只在功能柱内合成, 不会在柱外合成. 如上所述, 在实际的生理条件下, 柱内存在的 NO 的浓度是稳定的, 但这个浓度比激活 状态下的浓度要低得多(如上所述低 2 3 个数量级), 因此, 可以忽略柱外产生的 NO. 此外, 柱内的血管 内皮细胞也产生 NO, 同样认为这些 NO 的浓度与激 活状态下产生的浓度要低很多, 因此忽略血管和神 经元 NO 的对流运输.

（4）边界条件. $g(\mathrm{~d} c / \mathrm{d} x, \mathrm{~d} c / \mathrm{d} y, \mathrm{~d} c / \mathrm{d} z)=f(x, y, z)$.

(5) NO 增加过程用方波来模拟.

（6）整个大脑被假设为有足够大的空间使 NO 扩 散，因此可以被看作是无限的空间.

\section{4 设置 NO 的源强度}

由一般的扩散方程可知, 影响 NO 传播的主要因 素是源强度 (NO 的产生速度)和 $\mathrm{NO}$ 的固有参数(如扩 散系数 $D$ 和 $\mathrm{NO}$ 的半衰期)等. 仿真过程可将扩散系 数 $D$ 和 $\mathrm{NO}$ 的半衰期 $\left(t_{1 / 2}\right)$ 设为正常值, 然后改变其他 参数, 以详细讨论不同参数改变对 $\mathrm{NO}$ 在功能柱内扩 散的影响.

$S$ 值(每秒产生 NO 的浓度)可通过实验计算得出. 然而, 各种 $S$ 值在文献中已有报道 ${ }^{[23,24]}$. 这些差异可 能反映了不同 NO 产生速率和/或不同的 NO 测量方 法. 本模型关注由整个功能柱产生的浓度. 这个浓度
取决于特定皮层柱的激活程度, 因不同程度的激活 而不同. Buerk 等人 ${ }^{[25]}$ 已经计算出对大鼠后爪进行电 子刺激后大脑皮层产生的 $\mathrm{NO}$ 的生成速率. 在考虑文 献和本实验测量 NO 实验的基础上, 设置 $S$ 的常值为 $5000 \mathrm{nmol} \cdot \mathrm{L}^{-1} \cdot \mathrm{s}^{-1}$, 这意味着一串电刺激下功能柱内 $\mathrm{NO}$ 浓度达到的峰值为 $100 \mathrm{~ms}$ 内 $250 \mathrm{nmol} / \mathrm{L}$. 不同 $S$ 值的设定决定于它们对 NO 扩散的影响.

\section{2 结果}

\section{1 单个柱内单个 NO 脉冲产生时 NO 的扩散}

首先模拟最简单的 NO 扩散情况，也就是在单个 功能柱内, 一个 $\mathrm{NO}$ 脉冲产生时 $\mathrm{NO}$ 的扩散情况(参数 设置见图 2). 图 2 显示单个串刺激下产生一个 NO 脉 冲的情况. 图 2(A)显示了在超柱状体中 NO 扩散的一 个横截面, 二维扩散见图 2(B)(图中伪色彩表明 NO 的扩散浓度). 由图可见, 在中心点 $\mathrm{NO}$ 浓度高于参考 点(功能柱外的一点), 表明功能刺激下产生的 NO 主 要集中在激活区域的中心. 此属性使得 NO 作用具有 局部的特性.

关于 $\mathrm{NO}$ 在时间维度的扩散, 图 2(C)显示了 $\mathrm{NO}$ 在功能柱的中心点和参考点 (功能柱外的一点, 为一 个功能柱的距离)浓度剖面. 除了在参考点和中心点 不同程度的 $\mathrm{NO}$ 浓度, 还可以从此图观察到, $\mathrm{NO}$ 浓度 的持续高于某一给定的阈值(如图虚线所示)的时间, 在参考点和中心点分别是 0.4 和 $0.6 \mathrm{~s}$. 两种刺激 $\mathrm{NO}$ 反应时间均小于 $1 \mathrm{~s}$.

\section{2 不同刺激强度对 NO 扩散的影响}

刺激强度对 $\mathrm{NO}$ 扩散是最重要的影响因素. 接下 来研究单个被激活的皮质功能柱内刺激强度( $S$ 值)变 化对 NO 扩散的影响. 为清楚阐明 NO 扩散的时空动 力学, 定义了 2 个特征值来表征 $\mathrm{NO}$ 扩散的动态过程, 即“影响时间”和“影响半径”. “影响时间”是在给定区 域 NO 浓度在阈值浓度以上的持续时间, “影响半径” 被定义为在一定时间内 $\mathrm{NO}$ 浓度高于阈值浓度的范 围. 这 2 个特征值的生理学意义是动脉将在“影响时 间”和“影响半径”的范围内进行舒张，血流动响应过 程可用其描述.

在仿真过程中, 设置了不同的 $S$ 值, 而参数 $t_{1 / 2}$ 和系数 $D$ 是不变的. 结果表明, “影响半径”随着 $S$ 值 的增强而变大(图 3). $S$ 值在 $1000 \sim 5000 \mathrm{nmol} \cdot \mathrm{L}^{-1} \cdot \mathrm{s}^{-1}$ 范 
(A)
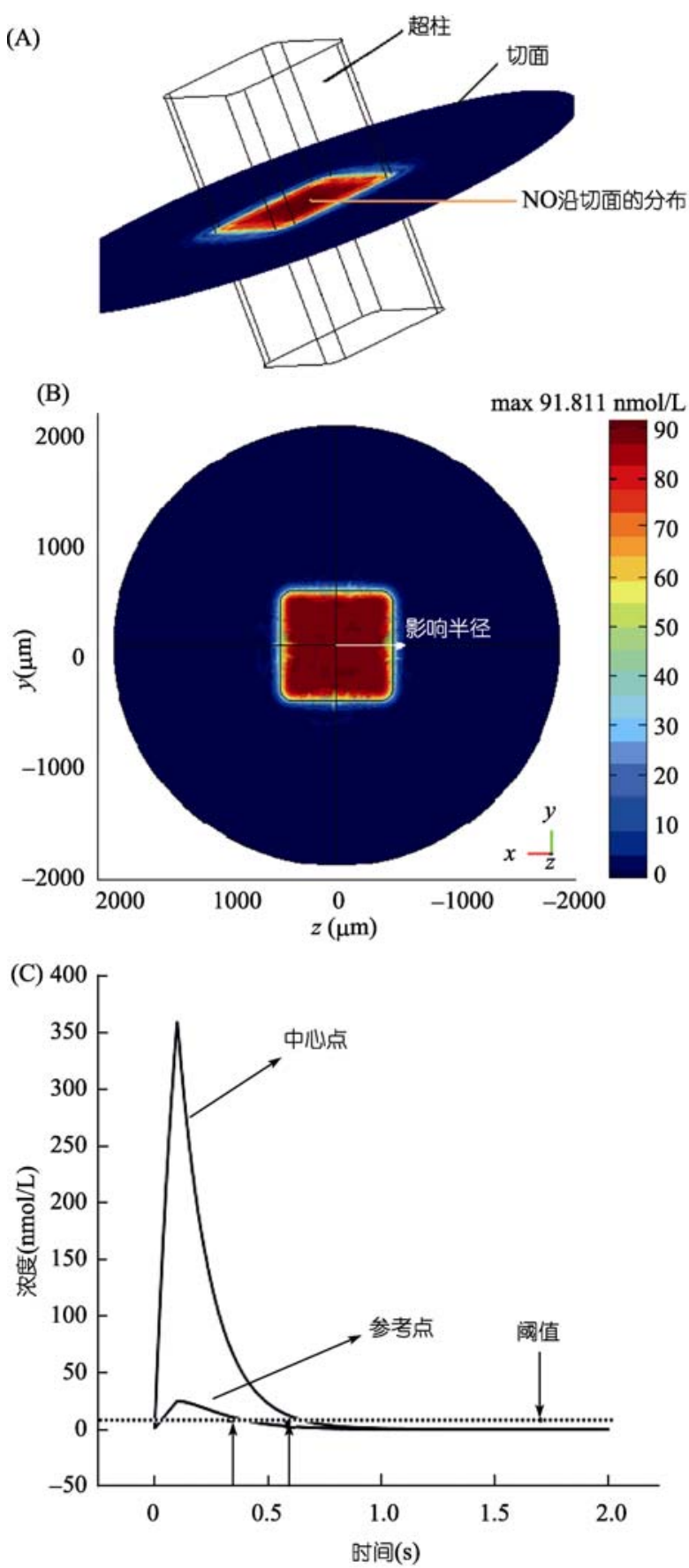

图 2 在一给定的激活超柱中 NO 扩散浓度的剖面图 (A) 显示了在超柱中 $\mathrm{NO}$ 扩散的横截面; (B) 显示了 $\mathrm{NO}$ 的二维扩 散(伪色彩表明 NO 扩散浓度: 红色表明饱和 NO 浓度, 黄色和绿 色以递减顺序表明较低的浓度; (C) 显示了在功能柱的中心点和 参考点 NO 浓度变化的时间过程. 虚线表示 NO 的阈值. 虚线箭头 表示在中心点和参考点 NO 浓度的持续时间持续高于阈值. 此处 使 $\mathrm{t}_{1 / 2}=100 \mathrm{~ms}$, NO 源生物合成时长为 $100 \mathrm{~ms}, D=3300 \mu \mathrm{m}^{2} / \mathrm{s}, S=$ $5000 \mathrm{nmol} \cdot \mathrm{L}^{-1} \cdot \mathrm{s}^{-1}$, 峰值浓度为 $361.21 \mathrm{nmol} / \mathrm{L}$
围内对“影响半径”的影响最大. 当 $S$ 值大于 5000 $\mathrm{nmol} \cdot \mathrm{L}^{-1} \cdot \mathrm{s}^{-1}$ 时影响变弱, 如当 $S$ 值从 5000 增强至 $25000 \mathrm{nmol} \cdot \mathrm{L}^{-1} \cdot \mathrm{s}^{-1}$ 时, “影响半径”只从 $837 \mu \mathrm{m}$ 增大到 $857 \mu \mathrm{m}$, 即脉冲强度增加了 5 倍, 而净“影响半径” $(857 \mu \mathrm{m}-837 \mu \mathrm{m}=20 \mu \mathrm{m})$ 增加很少. 该结果表明, NO 只对一个激活的功能柱内部或附近的动脉产生作用, 即 NO 的扩散范围是有限的、局部的，最大“影响半 径”小于 $900 \mu \mathrm{m}$. 因为功能柱半径为 $500 \mu \mathrm{m}$, 可以计 算出最大的净“影响半径”为 $400 \mu \mathrm{m}(900 \mu \mathrm{m}-500 \mu \mathrm{m})$ 表明, “影响半径”不会超越另一功能柱(这里为 500 $\mu \mathrm{m})$, 即使功能柱受到最强的刺激.
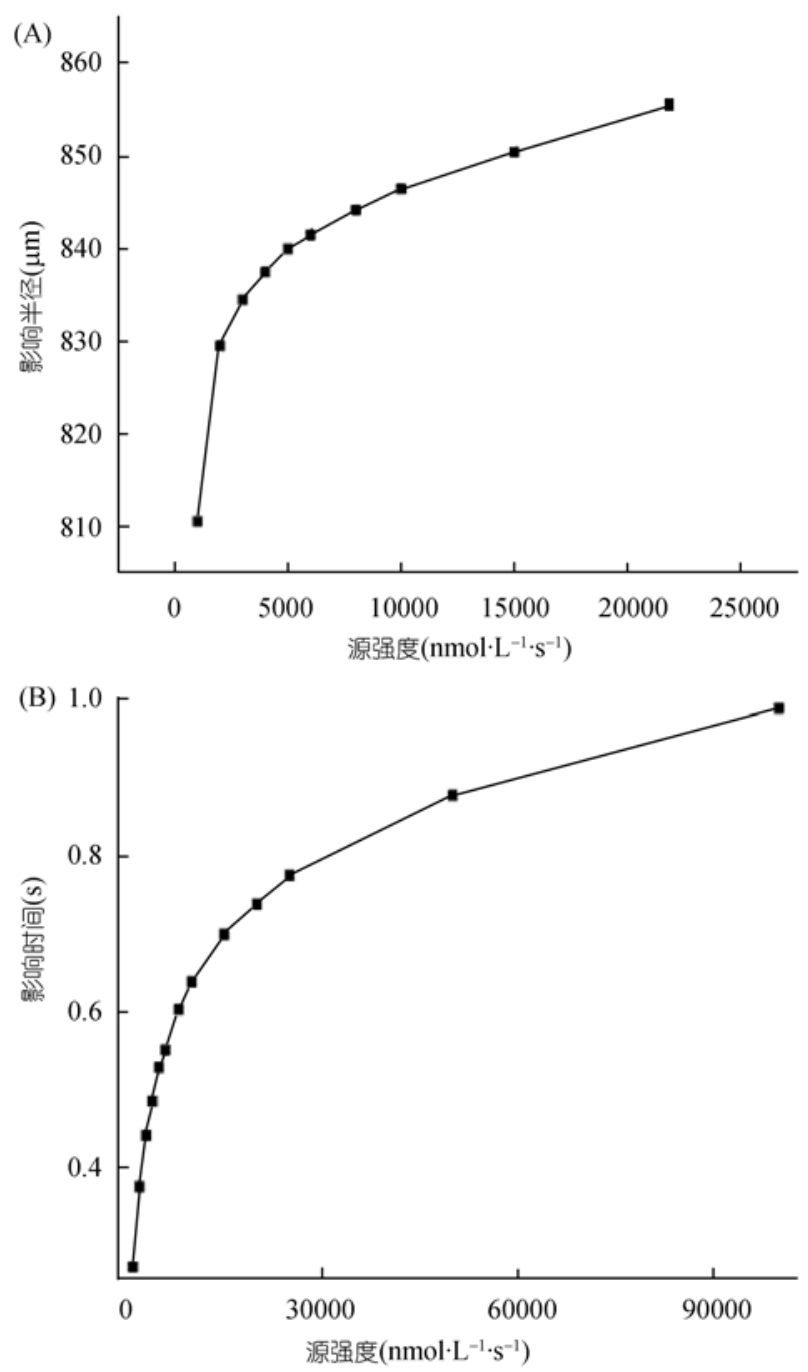

图 3 刺激强度变化对 NO 扩散的影响

(A) $S$ 值改变对“影响半径”的影响; (B) S 值改变对“影响时间”的影 响. $t_{1 / 2}=100 \mathrm{~ms}, D=3300 \mu \mathrm{m}^{2} / \mathrm{s}$, 极限值: $1 \mathrm{nmol} / \mathrm{L}$ 
“影响时间”随着 $S$ 值的增强而增加(图 3(B)). $S$ 值 在 $1000 \sim 3000 \mathrm{nmol} \cdot \mathrm{L}^{-1} \cdot \mathrm{s}^{-1}$ 范围内的变化导致的“影响 时间”增加要比 $S$ 值在 $3000 \mathrm{nmol} \cdot \mathrm{L}^{-1} \cdot \mathrm{s}^{-1}$ 以上范围大的 多. “影响时间” 将在 $S$ 值为 $9000 \mathrm{nmol} \cdot \mathrm{L}^{-1} \cdot \mathrm{s}^{-1}$ 时达到最 大并基本保持不变. 可以从曲线发现, “影响时间”的 上限约为或小于 $1 \mathrm{~s}$. 结果表明, $\mathrm{NO}$ 引起的皮质功能 柱内的血流动力学反应的持续时间小于 $1 \mathrm{~s}$.

\section{3 不同占空比对 NO 扩散的影响}

在生理学条件下, 刺激通常是系列串刺激而不 是一个单个的刺激. 为验证一个功能柱中系列串刺 激和单个刺激对 $\mathrm{NO}$ 的扩散行为是否有差异, 因此研 究系列串刺激对 NO 扩散的影响. 这里, 占空比这一 概念用于描述系列串刺激的过程. 刺激长度 (NO 产 生的方波)与整个持续时长的比例被称作占空比(脉 冲串长度/脉冲长度, 如 $200 \mathrm{~ms}$ 刺激时长/500 $\mathrm{ms}$ 脉冲 持续时长, 占空比 $=0.4)$. 研究占空比的变化对 $\mathrm{NO}$ 扩 散的影响. 图 4(B) 显示在中心点和参考点 NO 浓度随 占空比的增加而增加, 但是在中心点的增加程度远 大于参考点. 再一次表明, 在连续刺激下, $\mathrm{NO}$ 的作用 也是局部的.

图 4(C)给出当 $t=500 \mathrm{~ms}$ 时, 不同占空比情况下, $\mathrm{NO}$ 浓度分布剖面图(沿着过中心点的一条线). 浓度 分布图清楚地表明, 串刺激下 NO 浓度和“影响半径” 都大于单个刺激. 如当占空比为 0.1 时, 中心点的 NO 浓度约为 $13 \mathrm{nmol} / \mathrm{L}$, “影响半径”约为 $580 \mu \mathrm{m}$; 而当 占空比为 0.2 时, 相应的值分别为 $25 \mathrm{nmol} / \mathrm{L}$ 和 680 $\mu \mathrm{m}$. 结果表明, 连续刺激导致 $\mathrm{NO}$ 的浓度大大增加. 如果比较这两种刺激方法产生的 “影响半径”增加程 度(改变源强和改变占空比的方法), 可以发现, 改变 占空比导致的“影响半径”增加远高于改变源强度导 致的“影响半径”增加. 如当占空比从 0.1 增加至 0.2 时, “影响半径”的净增值约为 $100 \mu \mathrm{m}(680-580)$, 而当 源强从 5000 增加到 $25000 \mathrm{nmol} \cdot \mathrm{L}^{-1} \cdot \mathrm{s}^{-1}$ 时, 这个值仅 为 $50 \mu \mathrm{m}$. 据此可以推断, 生理学上, 增加刺激频率 较增加刺激强度可能更有效.

\section{4 激活区域范围变化对 NO 横向扩散的影响}

上述结果是只有一个功能柱被激活时的情况. 在实际生理情况下, 通常是多个功能柱被激活. 接下 来研究当多个功能柱被激活后 NO 的扩散. 基于图 5 中对几何结构的定义, 受激活皮层区的大小按几何 阵列排列为 $1 \times 1,2 \times 2,3 \times 3,4 \times 4$ 和 $5 \times 5$, 分别代表一个
区域为 $1,4,9,16,25$ 的功能柱. 不同的阵列边长分别 为 $1000,2000,3000,4000$ 和 $5000 \mu \mathrm{m}$. 这些边长代表 被激活脑区的大小. 这里假设在功能柱内氮能神经 元是均匀分布并且在功能柱内没有对流存在.
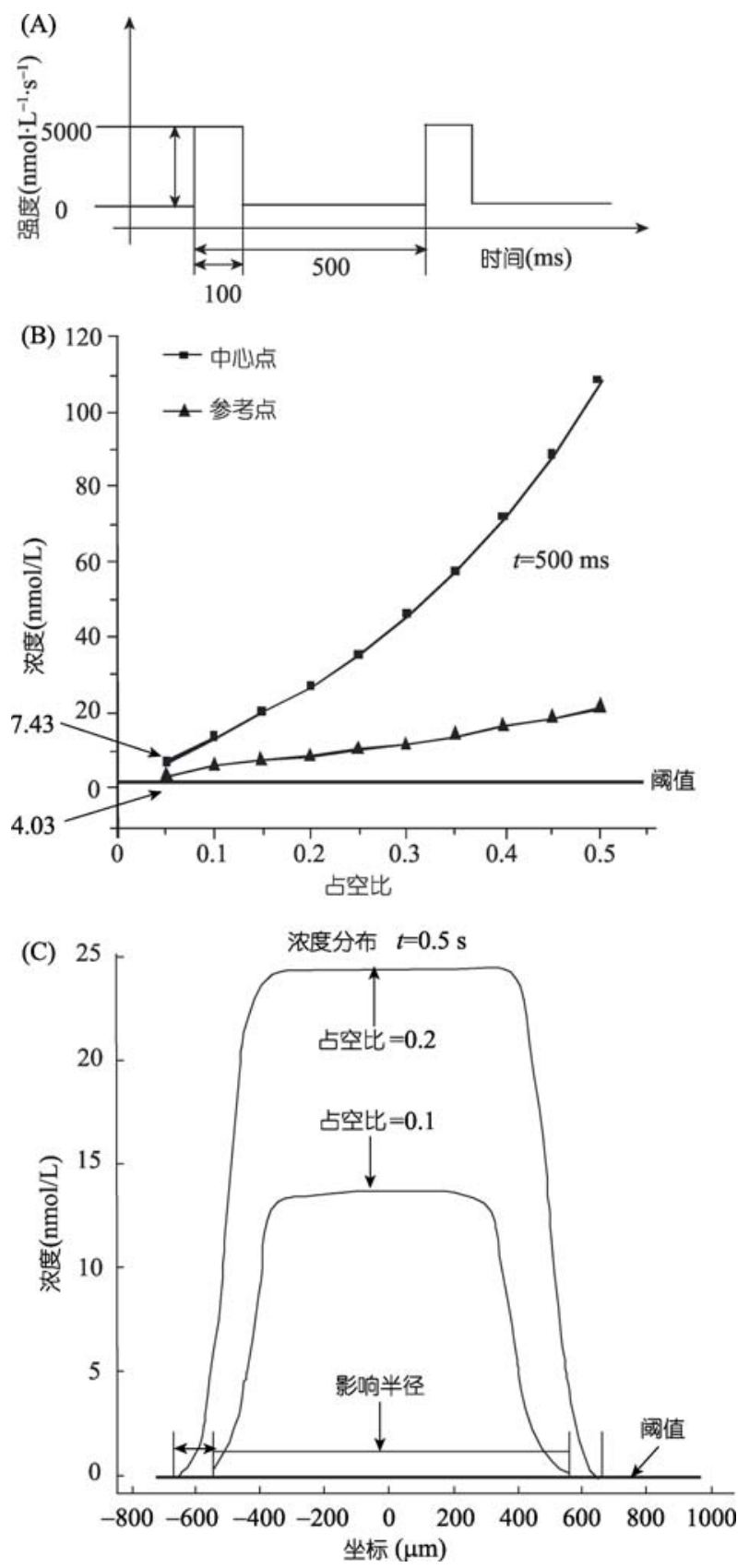

图 4 连续刺激下 NO 的扩散

(A) 占空比定义示意图; (B) 占空比在中心点和参考点对 NO 最高 浓度 $(t=500 \mathrm{~ms}$ 时在单个脉冲的末尾)的影响; (C) 在 $t=500 \mathrm{~ms}$ 时, 不 同占空比情况下, 沿着过中心点的一条线的 NO 浓度分布的剖面图. $t_{1 / 2}=100 \mathrm{~ms}, D=3300 \mu \mathrm{m}^{2} / \mathrm{s}$, 阈值: $1 \mathrm{nmol} / \mathrm{L}$ 
图 5 显示了激活区域大小与“影响半径”的关系. “影响半径”将随着边长增大而增大. 如边长为 1000 $\mu \mathrm{m} \times 1000 \mu \mathrm{m}$ 时, “影响半径”约为 $650 \mu \mathrm{m}$, 边长为 $2000 \mu \mathrm{m} \times 2000 \mu \mathrm{m}$ 时, 约 $1200 \mu \mathrm{m}$. 显然比较绝对值 的大小意义不大, 不如比较不同的阵列下“影响半 径”的净增加更有意义. 因此定义一个特征参数为 “延伸区”：对于一个给定的被激活的功能柱，有效的 “影响半径”减去功能柱边长. 比较结果如图 5(B)所示. 发现, 对于受到一个刺激的功能柱, “延伸区”约为 $150 \mu \mathrm{m}(650 \mu \mathrm{m}-500 \mu \mathrm{m})$, 然而当其为 $2000 \mu \mathrm{m} \times 2000$ $\mu \mathrm{m}$ 时, “延伸区” 为 $200 \mu \mathrm{m}$ (在一定刺激下). 显然, “延伸区”会随着被激活脑区的扩大而增大.

为进一步比较, 定义另一个称为“延伸比”的参 量(这里定义为对于一给定的被激活区域, 2 倍的延伸 区半径除以激活区边长)时, 可以发现“延伸比”将随 着边长的增加而减小 (图 5(C)). 如当大小为 1000 $\mu \mathrm{m} \times 1000 \mu \mathrm{m}$ 时, “延伸比”约为 0.6 , 当大小为 2000 $\mu \mathrm{m} \times 2000 \mu \mathrm{m}$ 时, 约为 0.55 . 结果表明, 被激活区域 的大小变化对 NO 扩散有影响, 但是影响有限.

\section{5 不同 NO 参数对 NO 扩散的影响}

Philippides 等人 ${ }^{[10]}$ 在模拟实验中深入研究了基 于神经丛形态的里 $\mathrm{NO}$ 半衰期 $t_{1 / 2}$ 和 $\mathrm{NO}$ 扩散系数 $D$ 对 NO 扩散的影响. 这里, 将专一地探讨在一个明确 形态的功能柱内这些参数变化对 $\mathrm{NO}$ 扩散行为的影响. $\mathrm{NO}$ 扩散系数 $D$, 在盐水溶液和类脂中约为 3300 $\mu \mathrm{m}^{2} / \mathrm{s}$. 然而, 一些研究表明, NO 并不像预期的像在 盐水溶液或血脂中那样几乎自由地在体内扩散. 因 此, 从 2 0.1 倍的标准值改变 $D\left(3300 \mu \mathrm{m}^{2} / \mathrm{s}\right)$. 结果表 明, 扩散系数 $D$ 没有影响“影响半径”(数据未显示), 但是影响“影响时间”. 同时发现, 扩散系数 $D$ 不仅影 响参照点, 而且其在功能柱内的影响是不变的(图 6(A)). 系数 $D$ 对“影响时间”最大的影响在 330 3300 $\mu \mathrm{m}^{2} / \mathrm{s}$ 范围内. 如上所述, $3300 \mu \mathrm{m}^{2} / \mathrm{s}$ 是在盐水的基础 上得到的. 系数 $D$ 在脑内的实际作用尚未被阐明, 但 是由于脑组织的高黏度性, 其值必定小于 3300 $\mu \mathrm{m}^{2} / \mathrm{s}$.

$\mathrm{NO}$ 的半衰期 $\left(t_{1 / 2}\right)$, 由文献[26,27]可知, $\mathrm{NO}$ 在体 内的半衰期值是不确定的, 取决于周围的反应物. 因 此, 设定从 $5 \mathrm{~s}$ 到 $0.1 \mathrm{~ms}$ 不同的 $t_{1 / 2}$ 值, 并观察在一个 被激活功能柱内 NO 扩散的结果. 对“影响时间”和 “影响半径”的影响分别见图 6(B)和(C). 结果表明,
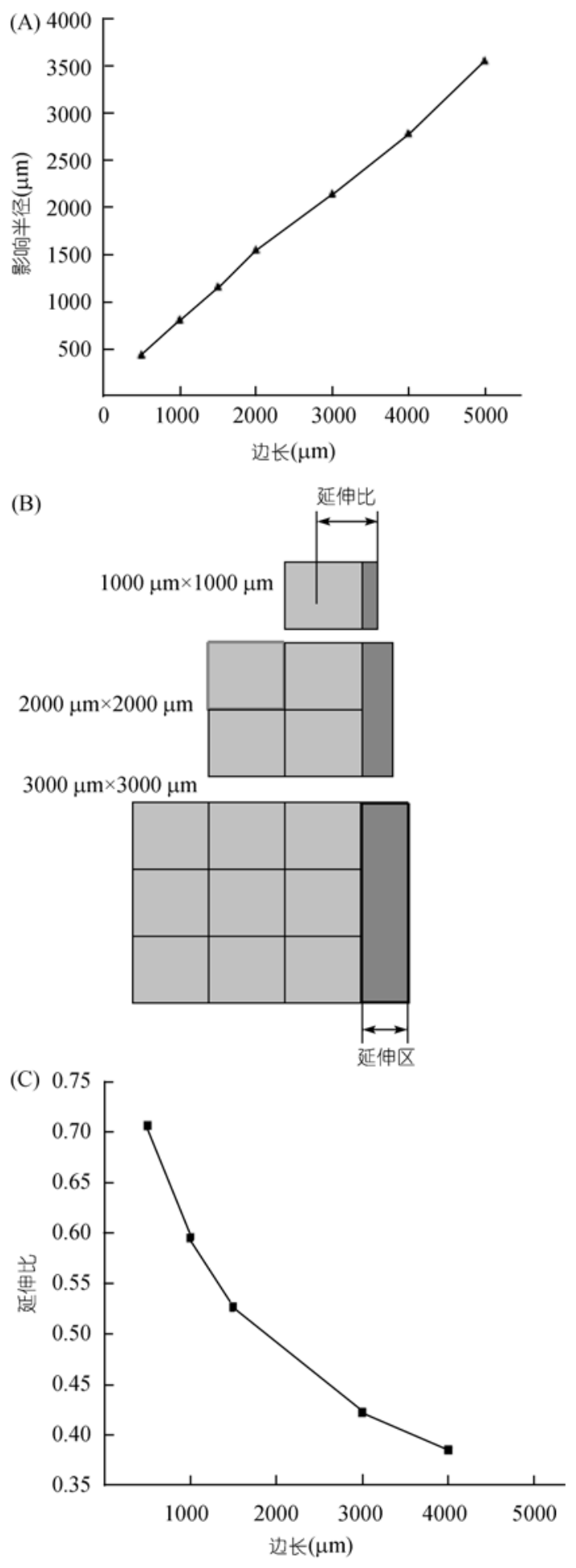

图 5 激活区域大小变化对 NO 横向扩散的影响 (A) “影响半径”随着被激活区域的增大而增大; (B) 表明“延伸区” 也随着被激活区域的增大而增大; (C) 表明了“延伸比”随着被激 活区域的增大而减小 

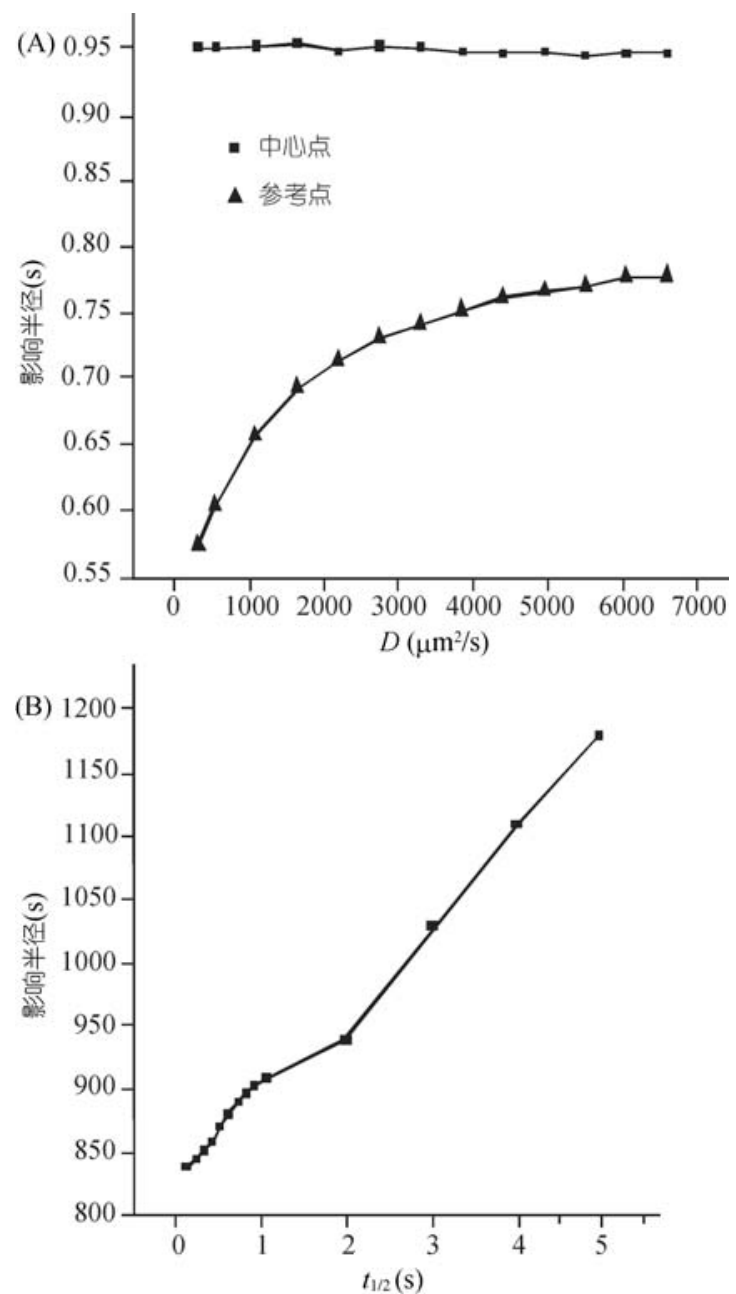

(C)

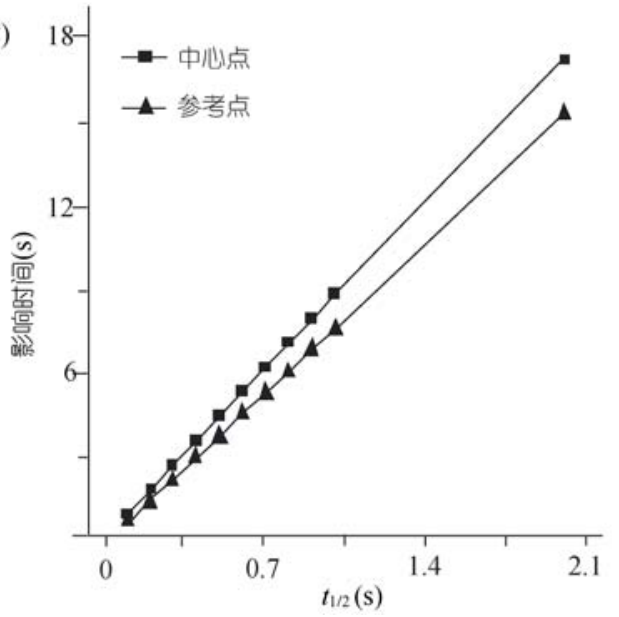

图 6 在被激活的功能柱内改变 NO 参量对 NO 扩散的影响 (A) 系数 $D$ 对 $\mathrm{NO}$ 扩散的影响 $\left(t_{1 / 2}=100 \mathrm{~ms}, S=5000 \mathrm{nmol} \cdot \mathrm{L}^{-1} \cdot \mathrm{s}^{-1}\right.$; (B) 和(C) 分别表示半衰期 $\left(t_{1 / 2}\right)$ 对 NO 扩散(“影响时间”和“影响半径”) 的影响 $t_{1 / 2}$ 对“影响时间”和“影响半径”的影响很大. 在研究 范围内发现了一个“影响时间”、“影响半径”和 $t_{1 / 2}$ 的 大致线性关系. $\mathrm{NO}$ 在脑组织中扩散时 $t_{1 / 2}$ 值是确定.

\section{3 讨论}

最近, 已经有一些研究团队运用成像技术在大 脑功能柱层面探讨神经-血管机制，如啮齿类的感觉 运动皮层功能柱、人的眼优势柱、猫的方向柱等 ${ }^{[28-30]}$. 本研究重点探讨了 $\mathrm{NO}$ 在功能柱水平扩散的时空动 态过程, 深入分析了在神经-血管偶联下动脉扩张中 作为主要控制因子 $\mathrm{NO}$ 的作用. 计算机模拟的结论是: 当脑区，如功能柱被激活后，功能性刺激产生的 NO 主要集中在功能柱中, 这一特性使 NO 在空间中的传 递是有限的. 因此，在神经-血管偶联中的调节脑血 流过程中, NO 的作用具有局部性.

Phillipiddes 等人 ${ }^{[10]}$ 也具体讨论了由脑神经丛产 生的 NO 在调节脑血流量中的可能性, 因而, 由此而 引出的问题是在脑血流调节中哪个形态(神经从或功 能柱)是更适合的作为脑血流调节的一种形态？解剖 学研究表明, 大脑皮层的神经纤维通常不与动脉相 联 ${ }^{[1]}$, 而在大脑功能柱内外分布着许多小动脉, 因此, 从解剖学角度来看, 功能柱的形态更为合适. 此外, 考虑在刺激区域中由 2 个不同形态(神经丛或功能柱) 产生的 NO 浓度, 由于在神经元细胞体和树突中 $\mathrm{nNOS}$ 合酶分布的不同, 大脑功能柱产生的 NO 浓度 可能比神经丛产生的要高 ${ }^{[31]}$. NO 浓度越高使得 NO 作用的距离越远、越有效. 综合考虑以上这两个方面, 同时考虑突触后产生的 $\mathrm{NO}$ 参与各种突触可塑性 ${ }^{[8,32]}$, 推测被激活的功能柱产生的 NO 可能被作为神经-血. 管偶联过程中的介导因子, 由神经丛产生的 NO 可能 在突触可塑性中扮演神经递质的角色.

对于在功能激活中 $\mathrm{NO}$ 信号作用的时间维度，本 模拟结果表明，NO 的有效浓度(阈值以上浓度)保持 在不到 $1 \mathrm{~s}$ 非常短的时间内. 然而, 激光多普勒研究 表明，在神经-血管过程中血流响应的峰值会保持几 秒. 怎样解释仿真模拟结果和激光多普勒测量结果 在时间维度上的不一致性？推测有两个主要原因导 致这种不一致性. 第一是在皮层表面的血流响应和 功能柱内的血流响应存在差异. 功能柱内的血流响 应是初级血流响应，而大脑表面的血流响应是次级 血流响应. 由激光多普勒检测到的血流量变化可能 
同时反映大脑表面和功能柱深层的终动脉的血流响 应信号. 在血液动力反应中初级和次级血流响应之 间可能有一次延迟或反应不一致. 另一个原因可能 是在 NO 之后存在其他血管活性因素或信号通路延长 血液动力反应. 这些因素或信号通路包括前列腺素 ${ }^{[33]}$, 其在神经-血管偶联过程中的作用最近被关注 ${ }^{[34]}$. 这 些不同的中介因素可能在神经-血管偶联的时间窗中
依次导致血流响应. 如 NO 可能在第一阶段直接产生 作用，持续时间约为 $1 \mathrm{~s}$; 脑血流短暂的增强不能满 足大脑营养和氧气的需求, 因此, 需要一个可以继续 调制的激励阶段; 前列腺素和星形胶质细胞可能在 这个阶段发挥作用. 这样一种假设需要更进一步的 研究去证实这些中介因素在神经-血管偶联中是否产 生顺序作用.

\section{参考文献}

1 Iadecola C. Regulation of the cerebral microcirculation during neural activity: is nitric oxide the missing link? Trends Neurosci, 1993 , 16: $206-214$

2 Knowles R G, Moncada S. Nitric oxide synthases in mammals. Biochem J, 1994, 298: 249-258

3 Iadecola C, Pelligrino D A, Moskowitz M A, et al. Nitric oxide synthase inhibition and cerebrovascular regulation. J Cereb Blood Flow Metab, 1994, 14: 175-192

4 Hayashi T, Katsumi Y, Inoue M, et al. The role of neuronal nitric oxide in the regional neurovascular coupling-Voxel-based comparison between perfusion and metabolic PET images. International Congress Series, 2002, 1235: 197-204

5 Gally J A, Montague P R, Jr Reeke G N. The NO hypothesis: possible effects of a short-lived, rapidly diffusible signal in the development and function of the nervous system. Proc Natl Acad Sci USA, 1998, 87: 3547-3551

6 Montague P R, Gally J A, Edelman G M. Spatial signaling in the development and function of neural connections. Cereb Cortex, 1991, 1: $199-220$

7 Wood J, Garthwaite J. Models of the diffusional spread of nitric oxide: implications for neural nitric oxide signaling and its pharmacological properties. Neuropharmacology, 1994, 33: 1235-1244

8 Garthwaite J, Boulton C L. Nitric oxide signaling in the central nervous system. Annu Rev Physiol, 1995, 57: 683-706

9 Philippides A, Husbands P, O’Shea M. Four-dimensional neuronal signaling by nitric oxide: a computational analysis. J Neurosci, 2000, 20: $1199-1207$

10 Philippides A, Ott S R, Husbands P A. et al. Modeling cooperative volume signaling in a plexus of nitric oxide synthase-expressing neurons. J neurosci, 2005, 25: 6520-6532

11 Estrada C, DeFelipe J. Nitric oxide-producing neurons in the neocortex: morphological and functional relationship with intraparenchymal microvasculature. Cereb Cortex, 1998, 8: 193-203

12 Hubel D H, Wiesel T N. Sequence regularity and geometry of orientation columns in the monkey striate cortex. J Comp Neurol, 1974, 158: $267-293$

13 Mountcastle V B. The columnar organization of the neocortex. Brain, 1997, 120: 701-722

14 Paxinos G, Watson C. The Rat Brain in Stereotaxic Coordinates. San Diego: Academic Press, 1998

15 Imura T, Kanatini S, Fukuta S. Layer-specific production of nitric oxide during cortical circuit formation in postnatal mouse brain. Cereb Cortex, 2005, 15: 332-340

16 Duvernoy H M, Delon S, Vannson J L. Cortical blood vessels of the human brain. Brain Res Bull, 1981, 7: 519-579

17 Woolsey T A, Rovainen C M, Cox S B. Neuronal units linked to microvascular modules in cerebral cortex: response elements for imaging the brain. Cereb Cortex, 1996, 6: 647-660

18 Crank J. The Mathematics of Diffusion. New York: Oxford University Press, 1980

19 Tirupathi R, Chandrupatla, Ashok D, et al. Introduction to Finite Elements in Engineering, 3rd ed. New York: McGraw-Hill Publishing Co. 1998

20 Artz J D, Toader V, Zavorin S I. In vitro activation of soluble guanylyl cyclase and nitric oxide release: comparison of NO donors and NO mimetics. Biochemistry, 2001, 40: 9256-9264

21 Mo E, Amin H, Bianco I H, et al. Kinetics of a cellular nitric oxide/cGMP/phosphodiesterase-5 pathway. J Biol Chem, 2004, 279: 2614926158

22 Griffiths C, Yamina B, Hall C. Nitric oxide inactivation in brain by a novel $\mathrm{O}_{2}$-dependent mechanism resulting in the formation of nitrate 
ions. Biochem J, 2002, 362: 459-464

23 Malinski T, Bailey F, Zhang Z G. Nitric oxide measured by a porphyrinic microsensor in rat brain after transient middle cerebral artery occlusion. J Cereb Blood Flow Metab, 1993, 13: 355-358

24 Shibuki K. An electrochemical microprobe for detecting nitric oxide release in brain tissue. Neurosci Res, 1990, 9: 69-76

25 Buerk D G, Ances B M, Greenberg J H. Temporal dynamics of brain tissue nitric oxide during functional forepaw stimulation in rats. NeuroImage, 2003, 18: 1-9

26 Griffiths C, Garthwaite J. The shaping of nitric oxide signals by a cellular sink. J Physiol, 2001, 536: 855-862

27 Thomas D T, Liu X, Kantrow S P. et al. The biological lifetime of nitric oxide: implications for the perivascular dynamics of $\mathrm{NO}$ and $\mathrm{O}_{2}$. Proc Natl Acad Sci USA, 2001, 98: 355-360

28 Devor A, Ulbert I, Dunn A K, et al. Coupling of the cortical hemodynamic response to cortical and thalamic neuronal activity. Proc Natl Acad Sci USA, 2005, 102: 3822-3827

29 Cheng K, Waggoner R A, Tanaka K. Human ocular dominance columns as revealed by high-field functional magnetic resonance imaging. Neuron, 2001, 32: 359-374

30 Duong T Q, Kim D S, Ugurbil K, et al. Localized cerebral blood flow response at submillimeter columnar resolution. Proc Natl Acad Sci USA, 2001, 98: 10904-10909

31 Yong Y, Ning G, Kutor J, et al. Nitric oxide spatial distribution in single cultured hippocampus neuron: investigation by projection of reconstructed 3-D image and visualization technique. Cell Biol Int, 2004, 28: 577-583

32 Hawkins R D, Son H, Arancio O. Nitric oxide as a retrograde messenger during long-term potentiation in hippocampus. Prog Brain Res, 1998, 118: 155-172

33 Niwa K, Araki E, Scott G, et al. Cyclooxygenase-2 contributes to functional hyperemia in whisker-barrel cortex. J Neurosci, 2000, 20: 763-770

34 Koehler R C, Gebremedhin, Harder D R. Role of astrocytes in cerebrovascular regulation. J Appl Physiol, 2006, 100: 307-317 